\title{
Effects of the $K^{+} \rightarrow \pi^{+} \nu \bar{\nu}$ and of other processes on the mixing hierarchies in the four-generation model
}

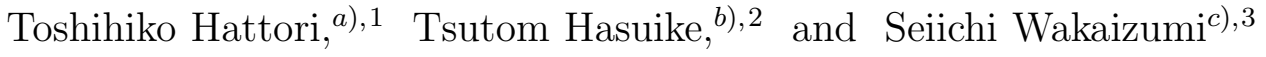 \\ a) Institute of Theoretical Physics, University of Tokushima, Tokushima 770-8502, Japan \\ b) Department of Physics, Anan College of Technology, Anan 774-0017, Japan \\ c) School of Medical Sciences, University of Tokushima, Tokushima 7r0-8509, Japan
}

\begin{abstract}
We analyze in the four-generation model the first measurement of the branching ratio of rare kaon decay $K^{+} \rightarrow \pi^{+} \nu \bar{\nu}$, along with the other processes of $K_{L}-K_{S}$ mass difference $\Delta m_{K}$, CP-violating parameter $\varepsilon_{K}, B_{d}-\bar{B}_{d}$ mixing, $B_{s}-\bar{B}_{s}$ mixing, $B\left(K_{L} \rightarrow \mu \bar{\mu}\right)$, and the upper bound values of $D^{0}-\bar{D}^{0}$ mixing and $B\left(K_{L} \rightarrow \pi^{0} \nu \bar{\nu}\right)$, and try to search for mixing of the fourth generation in the hierarchical mixing scheme of the Wolfenstein parametrization. Using the results for the mixing of the fourth generation, we discuss predictions of the $D^{0}-\bar{D}^{0} \operatorname{mixing}\left(\Delta m_{D}\right)$ and the branching ratio of directly CP-violating decay process $K_{L} \rightarrow \pi^{0} \nu \bar{\nu}$, and the effects on the $\mathrm{CP}$ asymmetry in neutral B meson decays and the unitarity triangle.
\end{abstract}

\footnotetext{
${ }^{1}$ e-mail: hattori@ias.tokushima-u.ac.jp

${ }^{2}$ e-mail: hasuike@anan-nct.ac.jp

${ }^{3}$ e-mail: wakaizum@medsci.tokushima-u.ac.jp
} 


\section{Introduction}

In the physics of quarks and leptons, it has been long since the Standard Model acquired a remarkable success. As is shown, however, in the issue of mass generation of quarks and leptons and the physics such as SUSY, physics beyond the Standard Model has become highly expected. In this direction, the flavor-changing neutral current(FCNC) processes play important roles through the one-loop effects for the search of additional Higgs, new gauge bosons, additional fermions, etc.

Here we focus on the new branching ratio of the FCNC process $K^{+} \rightarrow \pi^{+} \nu \bar{\nu}$, which is measured for the first time at the Brookhaven National Laboratory, $B=\left(4.2_{-3.5}^{+9.7}\right) \times 10^{-10}[$ [] . It should be remarked that the central value is 4-6 times larger than the Standard Model prediction, $B=(0.6-1.5) \times 10^{-10}[2]$, though the measurement is consistent with the theory within the experimental errors.

This process, $K^{+} \rightarrow \pi^{+} \nu \bar{\nu}$, had already been studied by Gaillard and Lee in 1974 and they obtained a branching ratio of $\sim 10^{-10}$ by using the short-distance $W-W$ box and $Z^{0}$-penguin diagrams in the "4-quark" model[[3]. After that in 1981, Inami and Lim derived the rigorous expressions for these and other related diagrams relevant to the FCNC processes and studied the effects of superheavy quarks and leptons in $K_{L} \rightarrow \mu \bar{\mu}, K^{+} \rightarrow \pi^{+} \nu \bar{\nu}$ and $K^{0}-\bar{K}^{0} \operatorname{mixing}[$ [⿴囗⿴囗十口], before the top-quark was discovered.

In this work, we analyze the new branching ratio of $K^{+} \rightarrow \pi^{+} \nu \bar{\nu}$ in the fourgeneration model[5] [6] [7] under the expectation that the above-mentioned factor 4-6 of the measured value relative to the Standard Model predictions may imply the existence of a fourth generation with roughly the same mixing as for the third generation. We will investigate various possible mixings for the fourth generation by imposing the constraints of $K^{+} \rightarrow \pi^{+} \nu \bar{\nu}$ and other processes of $K_{L}-K_{S}$ mass difference $\Delta m_{K}$, CP-violating parameter $\varepsilon_{K}, B_{d}-\bar{B}_{d}$ mixing, $B_{s}-\bar{B}_{s}$ mixing, $D^{0}-\bar{D}^{0}$ mixing, $B\left(K_{L} \rightarrow \pi^{0} \nu \bar{\nu}\right)$ and $B\left(K_{L} \rightarrow \mu \bar{\mu}\right)$, and we will study its effects 
on the $D^{0}-\bar{D}^{0}$ mixing and $B\left(K_{L} \rightarrow \pi^{0} \nu \bar{\nu}\right)$, of which only the upper bounds are experimentally known, $\mathrm{CP}$ violation in neutral $B$ meson decays and the unitarity triangle.

The paper is organized as follows. The four-generation model we use here is presented in Sec. II. In Sec. III we describe the phenomenological constraints on the model to search for possible mixings of the fourth generation. In Sec. IV we derive the "maximum" mixings allowed by the constraints. In Sec. V we discuss the consequences of the mixings on the $D^{0}-\bar{D}^{0}$ mixing and the branching ratio of another FCNC decay process $B\left(K_{L} \rightarrow \pi^{0} \nu \bar{\nu}\right)$, CP asymmetry in $B_{d}$ meson decays and the unitarity triangle, and finally we give conclusions.

\section{The four-generation model}

For the unitary $4 \times 4$ quark mixing matrix in the four-generation scheme, we will use the Hou-Soni-Steger parametrization [8]. The form of this parametrization is so complicated that we will not cite it here. It has, however, an advantage over the others that the third column and the fourth row have simple forms such that $\left(V_{u b}, V_{c b}, V_{t b}, V_{t^{\prime} b}\right)=\left(s_{z} c_{u} \mathrm{e}^{-\mathrm{i} \phi_{1}}, s_{y} c_{z} c_{u}, c_{y} c_{z} c_{u},-s_{u}\right)$ and $\left(V_{t^{\prime} d}, V_{t^{\prime} s}, V_{t^{\prime} b}, V_{t^{\prime} b^{\prime}}\right)=$ $\left(-c_{u} c_{v} s_{w} \mathrm{e}^{\mathrm{i} \phi_{3}},-c_{u} s_{v} \mathrm{e}^{\mathrm{i} \phi_{2}},-s_{u}, c_{u} c_{v} c_{w}\right)$, and $V_{u s}=s_{x} c_{z} c_{v}-s_{z} s_{u} s_{v} \mathrm{e}^{\mathrm{i}\left(\phi_{2}-\phi_{1}\right)}$, so that the three mixing angles $s_{x}\left(\equiv \sin \theta_{x}\right), s_{y}$ and $s_{z}$ give the elements $V_{u s}, V_{c b}$ and $V_{u b}$ respectively as in the Standard Model, and the phase $\phi_{1}$ corresponds to the Kobayashi-Maskawa(KM) CP-violating phase $\delta^{K M}[9]$. The angles $s_{u}\left(\equiv \sin \theta_{u}\right), s_{v}$ and $s_{w}$, which give the elements $V_{t^{\prime} b}, V_{t^{\prime} s}$ and $V_{t^{\prime} d}$ respectively, are new mixing angles, and $\phi_{2}$ and $\phi_{3}$ are new phases. $t^{\prime}$ and $b^{\prime}$ are the fourth generation up- and down-quark, respectively.

Since the magnitude of the three elements $V_{u s}, V_{c b}$ and $V_{u b}$ are experimentally determined from the semileptonic decays of hyperons, $B$ mesons to hadrons with $c$ - and $u$-quark, respectively, and are not affected by the existence of the fourth 
generation, we use the same values for the three angles $s_{x}, s_{y}$ and $s_{z}$ as in the Standard Model[2] as an input of our analysis;

$$
s_{x}=0.22, \quad s_{y}=0.040 \pm 0.003, \quad s_{z} / s_{y}=0.08 \pm 0.02,
$$

We search for the mixings of the fourth generation allowed by the experimental quantities related to various FCNC processes. The mixing among the three generations in the Standard Model is known to be hierarchical as is well expressed by the Wolfenstein parametrization [10],

$$
\begin{aligned}
V^{(3)} & =\left(\begin{array}{ccc}
V_{u d} & V_{u s} & V_{u b} \\
V_{c d} & V_{c s} & V_{c b} \\
V_{t d} & V_{t s} & V_{t b}
\end{array}\right) \\
& \simeq\left(\begin{array}{ccc}
1-\lambda^{2} / 2 & \lambda & A(\rho-\mathrm{i} \eta) \lambda^{3} \\
-\lambda & 1-\lambda^{2} / 2 & A \lambda^{2} \\
A(1-\rho-\mathrm{i} \eta) \lambda^{3} & -A \lambda^{2} & 1
\end{array}\right),
\end{aligned}
$$

where $\lambda \equiv \sin \theta_{C}(\simeq 0.22)$ is the expansion parameter in the Wolfenstein parametrization. In the spirit of this parametrization, we will study the following cases of the fourth generation mixing to derive a "maximum" one allowed by the abovementioned constraints;

$$
\begin{aligned}
\left(V_{t^{\prime}}, V_{t^{\prime} s}, V_{t^{\prime} b}, V_{t^{\prime} b^{\prime}}\right) \simeq \quad & \left(\lambda^{5}, \lambda^{4}, \lambda^{3}, 1\right), \\
& \left(\lambda^{4}, \lambda^{3}, \lambda^{2}, 1\right), \\
& \left(\lambda^{3}, \lambda^{2}, \lambda, 1\right), \\
& \left(\lambda^{2}, \lambda^{2}, \lambda, 1\right) \\
& \left(\lambda^{3}, \lambda^{2}, 1, \lambda\right), \\
& \left(\lambda^{2}, \lambda, 1, \lambda\right) \\
& \left(0, \lambda^{3}, \lambda, 1\right) \\
& \left(0, \lambda^{2}, \lambda, 1\right) .
\end{aligned}
$$

Here, we are not interested in the last two cases with $V_{t^{\prime} d}=0$, because we will focus on the factor 4-6 of the central value of the measured branching ratio of $K^{+} \rightarrow \pi^{+} \nu \bar{\nu}$, relative to the predicted value in the Standard Model. 
Table 1: Combinations of relevant mixing matrix elements for $\Delta m_{B_{d}}, b \rightarrow$ $s \gamma, K^{+} \rightarrow \pi^{+} \nu \bar{\nu}$ and $\left(K_{L} \rightarrow \mu \bar{\mu}\right)_{\mathrm{SD}}$ for the third generation in the Standard Model and the four cases of the fourth generation mixing.

\begin{tabular}{ccccc}
\hline \hline Mixing & $\Delta m_{B_{d}}$ & $b \rightarrow s \gamma$ & $K^{+} \rightarrow \pi^{+} \nu \bar{\nu}$ & $\left(K_{L} \rightarrow \mu \bar{\mu}\right)_{\mathrm{SD}}$ \\
\hline$\left(V_{t d}, V_{t s}, V_{t b}\right)$ & $V_{t d} V_{t b}$ & $V_{t s} V_{t b}$ & $V_{t d} V_{t s}$ & $V_{t d} V_{t s}$ \\
$\left(\lambda^{3}, \lambda^{2}, 1\right)$ & $\lambda^{3}$ & $\lambda^{2}$ & $\lambda^{5}$ & $\lambda^{5}$ \\
\hline$\left(V_{t^{\prime} d}, V_{t^{\prime} s}, V_{t^{\prime} b}\right)$ & $V_{t^{\prime} d} V_{t^{\prime} b}$ & $V_{t^{\prime} s} V_{t^{\prime} b}$ & $V_{t^{\prime} d} V_{t^{\prime} s}$ & $V_{t^{\prime} d} V_{t^{\prime} s}$ \\
$\left(\lambda^{5}, \lambda^{4}, \lambda^{3}\right)$ & $\lambda^{8}$ & $\lambda^{7}$ & $\lambda^{9}$ & $\lambda^{9}$ \\
$\left(\lambda^{4}, \lambda^{3}, \lambda^{2}\right)$ & $\lambda^{6}$ & $\lambda^{5}$ & $\lambda^{7}$ & $\lambda^{7}$ \\
$\left(\lambda^{3}, \lambda^{2}, \lambda\right)$ & $\lambda^{4}$ & $\lambda^{3}$ & $\lambda^{5}$ & $\lambda^{5}$ \\
$\left(\lambda^{2}, \lambda^{2}, \lambda\right)$ & $\lambda^{3}$ & $\lambda^{3}$ & $\lambda^{4}$ & $\lambda^{4}$ \\
\hline \hline
\end{tabular}

Table 1 shows the products of the relevant mixing matrix elements of the dominant contributions to the one-loop diagrams in $B_{d}-\bar{B}_{d}$ mixing $\left(\Delta m_{B_{d}}\right)$, $b \rightarrow s \gamma$ decay, $K^{+} \rightarrow \pi^{+} \nu \bar{\nu}$ decay and short-distance contributions to $K_{L} \rightarrow$ $\mu \bar{\mu}\left(\left(K_{L} \rightarrow \mu \bar{\mu}\right)_{\mathrm{SD}}\right)$ for the Standard Model and the four-generation model with the first four cases of mixing of eq.(3). As seen in Table 1, the first two cases of $\left(V_{t^{\prime} d}, V_{t^{\prime} s}, V_{t^{\prime} b}\right) \simeq\left(\lambda^{5}, \lambda^{4}, \lambda^{3}\right)$ and $\left(\lambda^{4}, \lambda^{3}, \lambda^{2}\right)$ give too small contributions to affect the branching ratio of $K^{+} \rightarrow \pi^{+} \nu \bar{\nu}$ and they also do not give any significant contributions to $\Delta m_{B_{d}}$ and $\left(K_{L} \rightarrow \mu \bar{\mu}\right)_{\mathrm{SD}}$. The third case of $\left(V_{t^{\prime} d}, V_{t^{\prime} s}, V_{t^{\prime} b}\right) \simeq$ $\left(\lambda^{3}, \lambda^{2}, \lambda\right)$ gives the same order of contributions to $K^{+} \rightarrow \pi^{+} \nu \bar{\nu}$ and $\left(K_{L} \rightarrow \mu \bar{\mu}\right)_{\mathrm{SD}}$ as in the Standard Model. It turns out that even this favorable case of $\left(\lambda^{3}, \lambda^{2}, \lambda\right)$ does not contribute to $b \rightarrow s \gamma$ so much as in the Standard Model, so we will not include the process $b \rightarrow s \gamma$ in the following numerical analysis. Although the fifth and sixth cases of $\left(V_{t^{\prime} d}, V_{t^{\prime} s}, V_{t^{\prime} b}, V_{t^{\prime} b^{\prime}}\right) \simeq\left(\lambda^{3}, \lambda^{2}, 1, \lambda\right)$ and $\left(\lambda^{2}, \lambda, 1, \lambda\right)$ of eq.(3) are interesting, these cases have proved not to lead to any favorable solutions in our numerical analysis.

\section{Constraints on the model}

The constraints we impose on the model to search for the fourth generation 
mixing are the following, $K_{L}-K_{S}$ mass difference $\Delta m_{K}=(3.522 \pm 0.016) \times 10^{-12}$ $\mathrm{MeV}$ [11], CP-violating parameter in the neutral kaon system $\varepsilon_{K}=(2.28 \pm 0.02) \times$ $10^{-3}$ [11], $\Delta m_{B_{d}}=(3.12 \pm 0.20) \times 10^{-10} \mathrm{MeV}$ [11] for $B_{d}-\bar{B}_{d}$ mixing, $B\left(K^{+} \rightarrow\right.$ $\left.\pi^{+} \nu \bar{\nu}\right)=\left(4.2_{-3.5}^{+9.7}\right) \times 10^{-10}$ [], $\Delta m_{B_{s}}>52.0 \times 10^{-10} \mathrm{MeV}$ [12] for $B_{s}-\bar{B}_{s}$ mixing, $\Delta m_{D}<1.4 \times 10^{-10} \mathrm{MeV}[13]$ for $D^{0}-\bar{D}^{0}$ mixing, $B\left(K_{L} \rightarrow \pi^{0} \nu \bar{\nu}\right)<5.8 \times$ $10^{-5}$ [14] and $B\left(K_{L} \rightarrow \mu \bar{\mu}\right)_{\mathrm{SD}}<2.2 \times 10^{-9}$, where the upper bound of the shortdistance(SD) contribution to $B\left(K_{L} \rightarrow \mu \bar{\mu}\right)$ is taken to be the value estimated by Bélanger and Geng[15]. As for the directly CP-violating parameter in the neutral kaon system $\varepsilon^{\prime} / \varepsilon$, the experimental values by the two groups at CERN and Fermilab deviated from each other by more than 2.4 standard deviations and recently $\mathrm{KTeV}$ at Fermilab has obtained a completely consistent value of $\operatorname{Re}\left(\varepsilon^{\prime} / \varepsilon\right)=(28.0 \pm 4.1) \times 10^{-4}$ [16] with the one by NA31 of $\operatorname{Re}\left(\varepsilon^{\prime} / \varepsilon\right)=(23 \pm 7) \times$ $10^{-4}\left[17\right.$. The formulation of $\varepsilon^{\prime} / \varepsilon$ in the four-generation model with appropriate QCD corrections is complicated and is out of scope in our paper [18. So, we will not include $\varepsilon^{\prime} / \varepsilon$ here.

Each of the above-mentioned eight constraints is described in the following. (i) $K_{L}-K_{S}$ mass difference, $\Delta m_{K}$

The short-distance part of $\Delta m_{K}$ comes from the well-known $W-W$ box diagram with $c, t$ and $t^{\prime}$ as internal quarks as shown in Fig.1 in the four-generation model and the contribution is expressed, for example, for the box diagram with two $c$-quarks as follows,

$$
\Delta m_{K}(c, c)=\frac{G_{F}^{2} M_{W}^{2}}{6 \pi^{2}} f_{K}^{2} B_{K} m_{K} \operatorname{Re}\left[\left(V_{c s} V_{c d}^{*}\right)^{2}\right] \eta_{c c}^{K} S\left(x_{c}\right),
$$

where $S(x)$ is the Inami-Lim box function [4], $x_{c} \equiv m_{c}^{2} / M_{W}^{2}, m_{c}$ being the charmquark mass, $\eta_{c c}^{K}$ is the QCD correction factor including the next-to-leading order effects, and $f_{K}$ and $B_{K}$ are the decay constant and the bag parameter of the kaon, respectively. By taking for these parameters the values of $m_{c}=1.3 \mathrm{GeV}$, $\eta_{c c}^{K}=1.38$ [2], $f_{K}=0.16 \mathrm{GeV}$ and $B_{K}=0.75 \pm 0.15$ [2], we obtain from the inputs 
of eq.(匠) the $(c, c)$ contribution as $\Delta m_{K}(c, c)=(2.6-3.9) \times 10^{-12} \mathrm{MeV}$, which is already consistent by itself with the measured value. Numerically, $(c, t)$ and $(t, t)$ contributions are very small in comparison with the $(c, c)$ contribution, so we take a constraint for the fourth-generation contributions to be

$$
\left|\frac{\Delta m_{K}\left(c, t^{\prime}\right)+\Delta m_{K}\left(t, t^{\prime}\right)+\Delta m_{K}\left(t^{\prime}, t^{\prime}\right)}{\Delta m_{K}(c, c)}\right|<1
$$

as a loose constraint, since there are a large amount of the long-distance contributions. In eq.(5), $\left(t^{\prime}, t^{\prime}\right)$ contribution, $\Delta m_{K}\left(t^{\prime}, t^{\prime}\right)$, is given as follows,

$$
\Delta m_{K}\left(t^{\prime}, t^{\prime}\right)=\frac{G_{F}^{2} M_{W}^{2}}{6 \pi^{2}} f_{K}^{2} B_{K} m_{K} \operatorname{Re}\left[\left(V_{t^{\prime} s} V_{t^{\prime} d}^{*}\right)^{2}\right] \eta_{t^{\prime} t^{\prime}}^{K} S\left(x_{t^{\prime}}\right),
$$

where $x_{t^{\prime}} \equiv m_{t^{\prime}}^{2} / M_{W}^{2}, m_{t^{\prime}}$ being the fourth-generation $t^{\prime}$ mass, and $S\left(x_{t^{\prime}}\right)$ can be approximated as $0.707 x_{t^{\prime}}^{0.82}$ for $130 \leq m_{t^{\prime}} \leq 1200 \mathrm{GeV} . \eta_{t^{\prime} t^{\prime}}^{K}$ is the QCD correction factor which is taken here to the leading order as

$$
\eta_{t^{\prime} t^{\prime}}^{K}=\left[\alpha_{s}\left(m_{c}\right)\right]^{6 / 27}\left[\frac{\alpha_{s}\left(m_{b}\right)}{\alpha_{s}\left(m_{c}\right)}\right]^{6 / 25}\left[\frac{\alpha_{s}\left(m_{t}\right)}{\alpha_{s}\left(m_{b}\right)}\right]^{6 / 23}\left[\frac{\alpha_{s}\left(m_{b^{\prime}}\right)}{\alpha_{s}\left(m_{t}\right)}\right]^{6 / 21}\left[\frac{\alpha_{s}\left(\mu_{t^{\prime}}\right)}{\alpha_{s}\left(m_{b^{\prime}}\right)}\right]^{6 / 19} .
$$

In eq.(7), $\alpha_{s}(m)$ is the running coupling constant in QCD and is expressed as

$$
\alpha_{s}(m)=\frac{4 \pi}{\beta_{0} \ln \left(m^{2} / \Lambda^{2}\right)},
$$

where $\Lambda$ is the QCD scale of $0.10 \mathrm{GeV}$ and $\beta_{0}=11-\frac{2}{3} N_{f}, N_{f}$ being the number of active quark flavors at the relevant energy scale, and $\mu_{t^{\prime}} \simeq O\left(m_{t^{\prime}}\right) . \eta_{t^{\prime} t^{\prime}}^{K}$ turns out to be 0.61 for $m_{c}=1.3 \mathrm{GeV}, m_{b}=4.4 \mathrm{GeV}, m_{t}=180 \mathrm{GeV}, m_{b^{\prime}}=370 \mathrm{GeV}$ and $m_{t^{\prime}}=400 \mathrm{GeV}$, the constraint on the fourth-generation quark masses being described at the end of this section. Similarly, $\Delta m_{K}\left(t, t^{\prime}\right)$ and $\Delta m_{K}\left(c, t^{\prime}\right)$ are expressed as

$$
\begin{aligned}
& \Delta m_{K}\left(t, t^{\prime}\right)=2 \frac{G_{F}^{2} M_{W}^{2}}{6 \pi^{2}} f_{K}^{2} B_{K} m_{K} \operatorname{Re}\left[V_{t s} V_{t d}^{*} V_{t^{\prime} s} V_{t^{\prime} d}^{*}\right] \eta_{t t^{\prime}}^{K} S\left(x_{t}, x_{t^{\prime}}\right), \\
& \Delta m_{K}\left(c, t^{\prime}\right)=2 \frac{G_{F}^{2} M_{W}^{2}}{6 \pi^{2}} f_{K}^{2} B_{K} m_{K} \operatorname{Re}\left[V_{c s} V_{c d}^{*} V_{t^{\prime} s} V_{t^{\prime} d}^{*}\right] \eta_{c t^{\prime}}^{K} S\left(x_{c}, x_{t^{\prime}}\right),
\end{aligned}
$$


where $S\left(x_{t}, x_{t^{\prime}}\right)$ is the Inami-Lim function for $W-W$ box diagram with $t$ - and $t^{\prime}$-quark in the internal line[[]] and the QCD correction factors $\eta_{t t^{\prime}}^{K}$ and $\eta_{c t^{\prime}}^{K}$ are taken as 0.5 and 0.6 , respectively.

(ii)CP-violating parameter in neutral kaon system, $\varepsilon_{K}$

The quantity $\varepsilon_{K}$ is expressed by the imaginary part of hadronic matrix element of the effective Hamiltonian with $\Delta S=2$ between $K^{0}$ and $\bar{K}^{0}$, to which the shortdistance contribution comes from the $W-W$ box diagram as in $\Delta m_{K}$. The box contributions with $c$ - and $t$-quark and with two $t$ quarks give the expressions of

$$
\begin{aligned}
& \varepsilon_{K}(c, t)=\frac{1}{\sqrt{2} \Delta m_{K}} \frac{G_{F}^{2} M_{W}^{2}}{6 \pi^{2}} f_{K}^{2} B_{K} m_{K} \operatorname{Im}\left[V_{c s} V_{c d}^{*} V_{t s} V_{t d}^{*}\right] \eta_{c t}^{K} S\left(x_{c}, x_{t}\right), \\
& \varepsilon_{K}(t, t)=\frac{1}{\sqrt{2} \Delta m_{K}} \frac{G_{F}^{2} M_{W}^{2}}{12 \pi^{2}} f_{K}^{2} B_{K} m_{K} \operatorname{Im}\left[\left(V_{t s} V_{t d}^{*}\right)^{2}\right] \eta_{t t}^{K} S\left(x_{t}\right) .
\end{aligned}
$$

If we take the QCD correction factors including the next-to-leading order as $\eta_{c t}^{K}=0.47$ and $\eta_{t t}^{K}=0.57$ [2], the dominant terms in the $(c, t)$ - and $(t, t)$-box contributions lead to $\varepsilon_{K}(c, t) \simeq 2.83 \times 10^{-3} B_{K} \sin \phi_{1}$ and $\varepsilon_{K}(t, t) \simeq 2.41 \times 10^{-3} B_{K} \sin \left(2 \phi_{1}\right)$ in the Standard Model, where $\phi_{1}$ is the CP-violating phase $\delta^{K M}$. Since the magnitude of these two contributions is already close to the measured value $\varepsilon_{K}=(2.28 \pm 0.02) \times 10^{-3}$ by taking into consideration the theoretical uncertainty in the bag parameter value, $B_{K}=0.75 \pm 0.15$, we take the constraint from $\varepsilon_{K}$ on the model that the sum of the contributions from $c, t$ and $t^{\prime}$ quarks should be within the $1 \sigma$ error of the measured value,

$$
\sum_{i, j=c, t, t^{\prime}, i \leq j} \varepsilon_{K}(i, j)=(2.28 \pm 0.02) \times 10^{-3} .
$$

(iii) $B_{d}-\bar{B}_{d}$ mixing, $\Delta m_{B_{d}}$

The mass difference between the two mass-eigenstates of $B_{d}-\bar{B}_{d}$ system is given by the $W-W$ box diagram, and the $(t, t)$-box contribution is expressed by

$$
\Delta m_{B_{d}}(t, t)=\frac{G_{F}^{2} M_{W}^{2}}{6 \pi^{2}} f_{B}^{2} B_{B} m_{B_{d}}\left|V_{t b} V_{t d}^{*}\right|^{2} \eta_{t t}^{B} S\left(x_{t}\right),
$$


where $f_{B}$ and $B_{B}$ are the decay constant and the bag parameter for $B_{d}$ meson, respectively, and $\eta_{t t}^{B}$ is the QCD correction factor including the next-to-leading order effects. By taking for these parameters the values of $\sqrt{B_{B}} f_{B}=(0.20 \pm 0.04)$ $\mathrm{GeV}$ and $\eta_{t t}^{B}=0.55[2]$ and by using the inputs of eq.(1), we obtain the $(t, t)$ contribution $\Delta m_{B_{d}}(t, t)=(1.75-3.95) \times 10^{-10} \mathrm{MeV}$ in the Standard Model. This value is consistent with the measured value, $\Delta m_{B_{d}}=(3.12 \pm 0.20) \times 10^{-10} \mathrm{MeV}[11]$. Since $(c, c)$ and $(c, t)$ contributions are numerically very small in comparison with the $(t, t)$ contribution, we take the constraint from $\Delta m_{B_{d}}$ on the model that the sum of the contributions from $t$ and $t^{\prime}$ should be within the $1 \sigma$ error of the measured value as follows,

$$
\begin{aligned}
\frac{G_{F}^{2} M_{W}^{2}}{6 \pi^{2}} f_{B}^{2} B_{B} m_{B_{d}} & \times\left|\left(V_{t b} V_{t d}^{*}\right)^{2} \eta_{t t}^{B} S\left(x_{t}\right)+\left(V_{t^{\prime} b} V_{t^{\prime} d}^{*}\right)^{2} \eta_{t^{\prime} t^{\prime}}^{B} S\left(x_{t^{\prime}}\right)+2 V_{t b} V_{t d}^{*} V_{t^{\prime} b} V_{t^{\prime} d}^{*} \eta_{t t^{\prime}}^{B} S\left(x_{t}, x_{t^{\prime}}\right)\right| \\
& =(3.12 \pm 0.20) \times 10^{-10} \mathrm{MeV},
\end{aligned}
$$

where we take for the QCD correction factor $\eta_{t^{\prime} t^{\prime}}^{B}$ the following expression to the leading order,

$$
\eta_{t^{\prime} t^{\prime}}^{B}=\left[\alpha_{s}\left(m_{t}\right)\right]^{6 / 23}\left[\frac{\alpha_{s}\left(m_{b^{\prime}}\right)}{\alpha_{s}\left(m_{t}\right)}\right]^{6 / 21}\left[\frac{\alpha_{s}\left(\mu_{t^{\prime}}\right)}{\alpha_{s}\left(m_{b^{\prime}}\right)}\right]^{6 / 19},
$$

which turns out to be 0.58 for the same set of parameter values as for $\eta_{t^{\prime} t^{\prime}}^{K}$. Another QCD correction factor $\eta_{t t^{\prime}}^{B}$ in eq.(15) is taken as 0.5.

(iv) $B\left(K^{+} \rightarrow \pi^{+} \nu \bar{\nu}\right)$

The short-distance contributions to the FCNC decay $K^{+} \rightarrow \pi^{+} \nu \bar{\nu}$ come from the $W-W$ box diagram and $Z^{0}$-penguin diagrams as shown in Fig.2 in the fourgeneration model. The expression for the contributions including the next-toleading order QCD effects is given by Buchalla and Buras [19] 20] in the Standard Model and are summarized in ref.2. We add to their expression of the branching ratio the contribution from $t^{\prime}$-quark exchange as follows,

$$
B\left(K^{+} \rightarrow \pi^{+} \nu \bar{\nu}\right)=\kappa_{+}\left|\frac{V_{c d} V_{c s}^{*}}{\lambda} P_{0}+\frac{V_{t d} V_{t s}^{*}}{\lambda^{5}} \eta_{t} X_{0}\left(x_{t}\right)+\frac{V_{t^{\prime} d} V_{t^{\prime}}^{*}}{\lambda^{5}} \eta_{t^{\prime}} X_{0}\left(x_{t^{\prime}}\right)\right|^{2},
$$


where $\kappa_{+}=4.57 \times 10^{-11}, P_{0}$ is the sum of charm contributions to the two diagrams including the next-to-leading order QCD corrections 20 and $X_{0}$ is the sum of the $W-W$ box and $Z^{0}$-penguin functions without QCD corrections calculated by Inami and Lim [4], the expressions of $P_{0}$ and $X_{0}$ being summarized in ref.2. In eq.(17), $\eta_{t}(=0.985)$ is the next-to-leading order QCD correction factor to the $t$ quark exchange [2] [19], and we take $\eta_{t^{\prime}}=1.0$ for $t^{\prime}$-exchange, since $\eta_{t}$ is almost 1.0 and the running distance for for the QCD corrections for $t^{\prime}$-exchange is shorter for $m_{t^{\prime}}>m_{t}$ than that for the $t$-exchange. The constraint is that the branching ratio of eq.(17) should be consistent with the measured value of branching ratio $B=\left(4.2_{-3.5}^{+9.7}\right) \times 10^{-10}[1]$, since the long-distance contribution is estimated to be very small $\left(B \sim 10^{-13}\right)$ [21]. We do not take into consideration the mixing effect in the leptonic sector.

(v) $B_{s}-\bar{B}_{s}$ mixing

The dominant contributions to $B_{s}-\bar{B}_{s}$ mixing are the $W-W$ box diagrams with $t$ and $t^{\prime}$ exchanges as in $B_{d}-\bar{B}_{d}$ mixing. We take the constraint that the sum of $(t, t),\left(t, t^{\prime}\right)$ and $\left(t^{\prime}, t^{\prime}\right)$ contributions to $\Delta m_{B_{s}}$ should be larger than the present experimental lower bound; $\Delta m_{B_{s}}>52.0 \times 10^{-10} \mathrm{MeV}$ [12], where $\Delta m_{B_{s}}$ is the mass difference of the two mass eigenstates of $B_{s}-\bar{B}_{s}$ system. The constraint is expressed as follows,

$$
\begin{aligned}
\frac{G_{F}^{2} M_{W}^{2}}{6 \pi^{2}} f_{B_{s}}^{2} B_{B_{s}} m_{B_{s}} & \times\left|\left(V_{t b} V_{t s}^{*}\right)^{2} \eta_{t t}^{B} S\left(x_{t}\right)+\left(V_{t^{\prime} b} V_{t^{\prime} s}^{*}\right)^{2} \eta_{t^{\prime} t^{\prime}}^{B} S\left(x_{t^{\prime}}\right)+2 V_{t b} V_{t s}^{*} V_{t^{\prime} b} V_{t^{\prime} s}^{*} \eta_{t t^{\prime}}^{B} S\left(x_{t}, x_{t^{\prime}}\right)\right| \\
& >52.0 \times 10^{-10} \mathrm{MeV} .
\end{aligned}
$$

We take the quantity $\sqrt{B_{B_{s}}} f_{B_{s}}$ to be equal to that for $B_{d}-\bar{B}_{d}$ mixing, and the QCD correction factors $\eta_{t t}^{B}, \eta_{t^{\prime} t^{\prime}}^{B}$ and $\eta_{t t^{\prime}}^{B}$ are equal to the ones for $B_{d}-\bar{B}_{d}$ mixing.

(vi) $D^{0}-\bar{D}^{0}$ mixing

The dominant contribution to $D^{0}-\bar{D}^{0}$ mixing in the four-generation model is the $W-W$ box diagram with fourth-generation down-quark $b^{\prime}$ exchange 22 as shown 
in Fig.3. We take the constraint that this contribution to the mass difference between the two mass-eigenstates of $D^{0}-\bar{D}^{0}$ system should be smaller than the present experimental upper bound [13], $\Delta m_{D}\left(b^{\prime}, b^{\prime}\right)<1.4 \times 10^{-10} \mathrm{MeV}$, since the Standard Model box contribution of two s-quarks exchange 23] and the longdistance contributions 24] are estimated to be three to four orders of magnitude smaller than the upper bound. The constraint is expressed as follows,

$$
\Delta m_{D}\left(b^{\prime}, b^{\prime}\right)=\frac{G_{F}^{2} M_{W}^{2}}{6 \pi^{2}} f_{D}^{2} B_{D} m_{D} \operatorname{Re}\left[\left(V_{c b^{\prime}}^{*} V_{u b^{\prime}}\right)^{2}\right] \eta_{b^{\prime} b^{\prime}}^{D} S\left(x_{b^{\prime}}\right)<1.4 \times 10^{-10} \mathrm{MeV}
$$

where $x_{b^{\prime}} \equiv m_{b^{\prime}}^{2} / M_{W}^{2}$. We take for the QCD correction factor $\eta_{b^{\prime} b^{\prime}}^{D}$ the following expression to the leading order,

$$
\eta_{b^{\prime} b^{\prime}}^{D}=\left[\alpha_{s}\left(m_{b}\right)\right]^{6 / 25}\left[\frac{\alpha_{s}\left(m_{t}\right)}{\alpha_{s}\left(m_{b}\right)}\right]^{6 / 23}\left[\frac{\alpha_{s}\left(\mu_{b^{\prime}}\right)}{\alpha_{s}\left(m_{t}\right)}\right]^{6 / 21}
$$

which is about 0.58 for $\mu_{b^{\prime}} \simeq m_{b^{\prime}}=370 \mathrm{GeV}, m_{t}=180 \mathrm{GeV}$ and $m_{b}=4.4 \mathrm{GeV}$. We tentatively take $f_{D} \sqrt{B_{D}}=0.2 \mathrm{GeV}$ in the following numerical analyses, since the numerical result of $\Delta m_{D}\left(b^{\prime}, b^{\prime}\right)$ is of the order of $10^{-12} \mathrm{MeV}$ for the range of $f_{D} \sqrt{B_{D}}=(0.1-0.3) \mathrm{GeV}$. Incidentally, the Standard Model prediction of $\Delta m_{D}$ is around $\left.10^{-14} \mathrm{MeV} 22\right]$.

(vii) $B\left(K_{L} \rightarrow \pi^{0} \nu \bar{\nu}\right)$

The process $K_{L} \rightarrow \pi^{0} \nu \bar{\nu}$ is the "direct" CP-violating decay [25] and the rate is expressed by the imaginary part of sum of the same $W-W$ box and $Z^{0}$-penguin diagram amplitudes as in $K^{+} \rightarrow \pi^{+} \nu \bar{\nu}[2]$, since the CP-conserving contribution is known to be very strongly suppressed[26]. Therefore, we take the constraint that the sum of $t$ and $t^{\prime}$ contributions to the branching ratio should be smaller than the experimental upper bound[14], $B\left(K_{L} \rightarrow \pi^{0} \nu \bar{\nu}\right)<5.8 \times 10^{-5}$. The constraint is expressed as

$$
\kappa_{L}\left(\frac{\operatorname{Im}\left(V_{t d} V_{t s}^{*}\right)}{\lambda^{5}} \eta_{t} X_{0}\left(x_{t}\right)+\frac{\operatorname{Im}\left(V_{t^{\prime} d} V_{t^{\prime} s}^{*}\right)}{\lambda^{5}} \eta_{t^{\prime}} X_{0}\left(x_{t^{\prime}}\right)\right)^{2}<5.8 \times 10^{-5}
$$


where $\kappa_{L}=1.91 \times 10^{-10}, X_{0}$ is the same function and $\eta_{t}$ and $\eta_{t^{\prime}}$ are the same QCD correction factors as appeared in eq.(17) for $K^{+} \rightarrow \pi^{+} \nu \bar{\nu}$.

(viii) $B\left(K_{L} \rightarrow \mu \bar{\mu}\right)_{\mathrm{SD}}$

The process $K_{L} \rightarrow \mu \bar{\mu}$ is the CP-conserving decay. The short-distance(SD) contribution is given by the $W-W$ box and $Z^{0}$-penguin diagrams and the branching ratio for this part is expressed as [2]

$B\left(K_{L} \rightarrow \mu \bar{\mu}\right)_{\mathrm{SD}}=\kappa_{\mu}\left[\frac{\operatorname{Re}\left(V_{c d} V_{c s}^{*}\right)}{\lambda} P_{0}^{\prime}+\frac{\operatorname{Re}\left(V_{t d} V_{t s}^{*}\right)}{\lambda^{5}} \eta_{t}^{Y} Y_{0}\left(x_{t}\right)+\frac{\operatorname{Re}\left(V_{t^{\prime} d} V_{t^{\prime} s}^{*}\right)}{\lambda^{5}} \eta_{t^{\prime}}^{Y} Y_{0}\left(x_{t^{\prime}}\right)\right]^{2}$,

where $\kappa_{\mu}=1.68 \times 10^{-9}, P_{0}^{\prime}$ is the sum of charm contributions to the two diagrams including the next-to-leading order QCD corrections 20] and $Y_{0}$ the sum of the $W-W$ box and $Z^{0}$-penguin functions without QCD corrections calculated by Inami and $\operatorname{Lim}[4]$, the expressions of $P_{0}^{\prime}$ and $Y_{0}$ being summarized in ref.2. In eq.(22), $\eta_{t}^{Y}(=1.026)$ is the next-to-leading order QCD correction factor to the $t$ quark exchange [2] [19] and we take $\eta_{t^{\prime}}^{Y}=1.0$ for $t^{\prime}$ exchange for the same reason as stated for $K^{+} \rightarrow \pi^{+} \nu \bar{\nu}$. We take the constraint that the branching ratio of eq.(22) should be smaller than the upper bound of the short-distance contribution 15 as stated before at the beginning of this section, $B\left(K_{L} \rightarrow \mu \bar{\mu}\right)_{\mathrm{SD}}<2.2 \times 10^{-9}$. We do not take into consideration the mixing effect in the leptonic sector.

For the masses of $t^{\prime}$ and $b^{\prime}$, there is a constraint from $\rho$ parameter. If we denote the parameter $\rho_{0}$ as

$$
\rho_{0}=\frac{M_{W}^{2}}{M_{Z}^{2} \cos ^{2} \theta_{W} \hat{\rho}}
$$

where $\sin ^{2} \theta_{W}$ is the Weinberg angle and $\hat{\rho}$ is the quantity $M_{W}^{2} /\left(M_{Z}^{2} \cos ^{2} \theta_{W}\right)$, which involves the radiative correction effects from Higgs doublets and top-quark mass, then $\rho_{0}-1$ describes new sources of $\mathrm{SU}(2)$ breaking. The fourth generation makes $\rho_{0}$ deviate from 1 as 27

$$
\rho_{0}=1+\frac{3 G_{F}}{8 \sqrt{2} \pi^{2}}\left(m_{t^{\prime}}^{2}+m_{b^{\prime}}^{2}-\frac{4 m_{t^{\prime}}^{2} m_{b^{\prime}}^{2}}{m_{t^{\prime}}^{2}-m_{b^{\prime}}^{2}} \ln \frac{m_{t^{\prime}}}{m_{b^{\prime}}}\right) .
$$


The value of $\rho_{0}$ is now $\rho_{0}=0.9998 \pm 0.0008$ [27], and this constrains the masses of $t^{\prime}$ and $b^{\prime}$.

\section{Possible mixings of fourth generation}

We search for possible mixings of the fourth generation allowed by the eight constraints in the previous section by testing the typical hierarchical mixings of eq.(3) with the intention to obtain the "maximum" mixing compatible with the considerably large branching ratio of the rare decay $K^{+} \rightarrow \pi^{+} \nu \bar{\nu}$ with a factor of 4-6 as compared with the predictions in the Standard Model. From this point of view, the last two cases with $V_{t^{\prime} d}=0$ of eq.(3) are not interesting here.

Free parameters are the three phases $\phi_{1}, \phi_{2}$ and $\phi_{3}$ of the $4 \times 4$ mixing matrix. As for the masses of the fourth generation quarks, we choose tentatively $\left(m_{t^{\prime}}, m_{b^{\prime}}\right)=(400,370),(800,770)$ and $(1200,1170) \mathrm{GeV}$ as typical ones, which are compatible with the constraint of eq.(24). We vary the three phases in the range of $0 \leq \phi_{1}, \phi_{2}, \phi_{3} \leq 2 \pi$. We found no solutions compatible with the eight constraints for the exotic fifth and sixth cases of $\left(V_{t^{\prime} d}, V_{t^{\prime} s}, V_{t^{\prime} b}, V_{t^{\prime} b^{\prime}}\right) \simeq\left(\lambda^{3}, \lambda^{2}, 1, \lambda\right)$ and $\left(\lambda^{2}, \lambda, 1, \lambda\right)$ of eq.(3). So, we focus on the first four cases of eq.(3) here.

Strong constraints come from $\Delta m_{K}, \varepsilon_{K}, B_{d}-\bar{B}_{d}$ mixing, $K^{+} \rightarrow \pi^{+} \nu \bar{\nu}$ and $\left(K_{L} \rightarrow \mu \bar{\mu}\right)_{\mathrm{SD}}$. In the Standard Model, the largest contribution comes from the top-quarks for $B_{d}-\bar{B}_{d}$ mixing, $K^{+} \rightarrow \pi^{+} \nu \bar{\nu}$ and $\left(K_{L} \rightarrow \mu \bar{\mu}\right)_{\mathrm{SD}}$, and there the combination of the relevant CKM matrix elements is $V_{t d} V_{t b} \sim \lambda^{3}$ for $B_{d}-\bar{B}_{d}$ mixing and $V_{t d} V_{t s} \sim \lambda^{5}$ for $K^{+} \rightarrow \pi^{+} \nu \bar{\nu}$ and $\left(K_{L} \rightarrow \mu \bar{\mu}\right)_{\mathrm{SD}}$. On the other hand, the combinations of the corresponding matrix elements for $t^{\prime}$-quark are shown in Table 1 for each of the above four cases. By comparing these combinations between the Standard Model and the four-generation model, the numerical analyses give the following results; the cases of $\left(s_{w}, s_{v}, s_{u}\right)\left(\simeq\left(\left|V_{t^{\prime} d}\right|,\left|V_{t^{\prime} s}\right|,\left|V_{t^{\prime} b}\right|\right)\right)=\left(\lambda^{5}, \lambda^{4}, \lambda^{3}\right)$ and $\left(\lambda^{4}, \lambda^{3}, \lambda^{2}\right)$ give almost the same predictions to the above-mentioned eight 
quantities as in the Standard Model, since the contributions of the fourth generation are very small, as seen from Table 1 . For the case of $\left(\lambda^{3}, \lambda^{2}, \lambda\right)$, almost all the quantities satisfy the constraints with only one exception of $B\left(K_{L} \rightarrow \mu \bar{\mu}\right)_{\mathrm{SD}}$, for which this mixing gives a value several times larger than the upper bound. The last case of $\left(\lambda^{2}, \lambda^{2}, \lambda\right)$ predicts too large values for $B\left(K^{+} \rightarrow \pi^{+} \nu \bar{\nu}\right)$ and $B\left(K_{L} \rightarrow \mu \bar{\mu}\right)_{\mathrm{SD}}$. These results imply that the mixing of $\left(\lambda^{3}, \lambda^{2}, \lambda\right)$ is a little large for the fourth generation and it turns out that a mixing with $s_{w}$ and $s_{v}$ reduced by $20 \%$, that is, $\left(s_{w}, s_{v}, s_{u}\right)=\left(0.8 \lambda^{3}, 0.8 \lambda^{2}, \lambda\right)$ satisfies all of the eight constraints for $\left(m_{t^{\prime}}, m_{b^{\prime}}\right)=(400,370) \mathrm{GeV}$, the one with $s_{w}$ and $s_{v}$ reduced by $50 \%$, that is, $\left(s_{w}, s_{v}, s_{u}\right)=\left(0.5 \lambda^{3}, 0.5 \lambda^{2}, \lambda\right)$ satisfies them for $\left(m_{t^{\prime}}, m_{b^{\prime}}\right)=(800,770) \mathrm{GeV}$ and the one with $\left(s_{w}, s_{v}, s_{u}\right)=\left(0.3 \lambda^{3}, 0.3 \lambda^{2}, \lambda\right)$ does for $\left(m_{t^{\prime}}, m_{b^{\prime}}\right)=(1200,1170) \mathrm{GeV}$ as a maximum mixing. This strong energy-dependence of the reduction factors $\left(s_{w} / \lambda^{3}, s_{v} / \lambda^{2}, s_{u} / \lambda\right)$ is valid and reasonable, because the contribution of the $t^{\prime}$ quark exchange to the decay amplitudes of both $K^{+} \rightarrow \pi^{+} \nu \bar{\nu}$ and $K_{L} \rightarrow \pi^{0} \nu \bar{\nu}$ is proportional to $V_{t^{\prime} d} V_{t^{\prime} s}^{*} X_{0}\left(x_{t^{\prime}}\right) \simeq \frac{1}{8} s_{w} s_{v}\left(m_{t^{\prime}} / M_{W}\right)^{2} \mathrm{e}^{\mathrm{i}\left(\phi_{3}-\phi_{2}\right)}$, the one to the amplitude of $\left(K_{L} \rightarrow \mu \bar{\mu}\right)_{\mathrm{SD}}$ is $\operatorname{Re}\left(V_{t^{\prime} d} V_{t^{\prime} s}^{*}\right) Y_{0}\left(x_{t^{\prime}}\right) \simeq \frac{1}{8} s_{w} s_{v}\left(m_{t^{\prime}} / M_{W}\right)^{2} \cos \left(\phi_{3}-\phi_{2}\right)$, the contribution to $\Delta m_{K}$ is $\operatorname{Re}\left[\left(V_{t^{\prime} d}^{*} V_{t^{\prime} s}\right)^{2}\right] S\left(x_{t^{\prime}}\right) \simeq 0.707 s_{w}^{2} s_{v}^{2}\left(m_{t^{\prime}} / M_{W}\right)^{1.64} \cos 2\left(\phi_{2}-\right.$ $\left.\phi_{3}\right)$, and the one to $\Delta m_{B_{d}}$ is $\left|V_{t^{\prime} d}^{*} V_{t^{\prime} b}\right|^{2} S\left(x_{t^{\prime}}\right) \simeq 0.707 s_{w}^{2} s_{u}^{2}\left(m_{t^{\prime}} / M_{W}\right)^{1.64}$.

We show several typical solutions with respect to the three phases $\left(\phi_{1}, \phi_{2}, \phi_{3}\right)$ for the maximum mixing $\left(s_{w}, s_{v}, s_{u}\right)=\left(0.8 \lambda^{3}, 0.8 \lambda^{2}, \lambda\right)$ in the case of $\left(m_{t^{\prime}}, m_{b^{\prime}}\right)=$ $(400,370) \mathrm{GeV}$ in Table 2 , the ones for $\left(s_{w}, s_{v}, s_{u}\right)=\left(0.5 \lambda^{3}, 0.5 \lambda^{2}, \lambda\right)$ in the case of $\left(m_{t^{\prime}}, m_{b^{\prime}}\right)=(800,770) \mathrm{GeV}$ in Table 3 and the ones for $\left(s_{w}, s_{v}, s_{u}\right)=\left(0.3 \lambda^{3}, 0.3 \lambda^{2}, \lambda\right)$ in the case of $\left(m_{t^{\prime}}, m_{b^{\prime}}\right)=(1200,1170) \mathrm{GeV}$ in Table 4 . The values of $\left(\phi_{1}, \phi_{2}, \phi_{3}\right)$ allowed by the constraints constitute a certain region in the plane, surrounding each of the solutions in Tables 2, 3 and 4. In the Tables, we also give the predictions of $B\left(K^{+} \rightarrow \pi^{+} \nu \bar{\nu}\right), \Delta m_{D}, B\left(K_{L} \rightarrow \pi^{0} \nu \bar{\nu}\right)$, and the CP asymmetry for $B_{d} \rightarrow J / \psi K_{S}$, which is explained in the following section, for each of the solutions. 
Table 2: Typical solutions for the fourth-generation mixing $\left(s_{w}, s_{v}, s_{u}\right)=$ $\left(0.8 \lambda^{3}, 0.8 \lambda^{2}, \lambda\right)$ in case of $\left(m_{t^{\prime}}, m_{b^{\prime}}\right)=(400,370) \mathrm{GeV}$ for the three phases of $\left(\phi_{1}, \phi_{2}, \phi_{3}\right)$. Predictions of $B\left(K^{+} \rightarrow \pi^{+} \nu \bar{\nu}\right)\left(10^{-10}\right), \Delta m_{D}\left(\operatorname{in} 10^{-12} \mathrm{MeV}\right), B\left(K_{L} \rightarrow\right.$ $\left.\pi^{0} \nu \bar{\nu}\right)\left(10^{-10}\right)$ and the CP asymmetry for $B_{d} \rightarrow J / \psi K_{S}$ are added.

\begin{tabular}{c|c|c|c|c|c|c}
\hline \hline$\phi_{1}$ & $\phi_{2}$ & $\phi_{3}$ & $K^{+} \rightarrow \pi^{+} \nu \bar{\nu}$ & $\Delta m_{D}$ & $K_{L} \rightarrow \pi^{0} \nu \bar{\nu}$ & $C_{f}\left(B_{d} \rightarrow J / \psi K_{S}\right)$ \\
\hline $3 \pi / 2$ & $\pi / 6$ & $\pi / 2$ & 2.8 & 0.8 & 11.7 & -0.37 \\
$\pi / 4$ & $\pi / 3$ & 0 & 2.2 & 1.8 & 8.7 & 0.24 \\
$3 \pi / 2$ & $\pi / 2$ & $\pi / 4$ & 2.3 & 1.6 & 8.6 & -0.34 \\
$7 \pi / 12$ & $\pi / 2$ & $3 \pi / 4$ & 4.1 & 0.6 & 16.7 & 0.26 \\
$\pi / 2$ & $5 \pi / 6$ & $\pi / 2$ & 1.7 & 0.7 & 7.0 & 0.25 \\
$3 \pi / 4$ & $\pi$ & $3 \pi / 4$ & 1.5 & 0.8 & 5.8 & 0.17 \\
$13 \pi / 8$ & $\pi$ & $3 \pi / 4$ & 2.7 & 0.7 & 9.9 & -0.35 \\
$\pi / 2$ & $7 \pi / 6$ & $11 \pi / 12$ & 1.7 & 0.4 & 6.6 & 0.30 \\
$\pi / 3$ & $4 \pi / 3$ & $13 \pi / 12$ & 2.1 & 0.4 & 8.0 & 0.32 \\
$\pi / 2$ & $2 \pi$ & $7 \pi / 4$ & 1.6 & 2.0 & 6.2 & 0.34 \\
\hline
\end{tabular}

Table 3: Typical solutions for the fourth-generation mixing $\left(s_{w}, s_{v}, s_{u}\right)=$ $\left(0.5 \lambda^{3}, 0.5 \lambda^{2}, \lambda\right)$ in case of $\left(m_{t^{\prime}}, m_{b^{\prime}}\right)=(800,770) \mathrm{GeV}$ for the three phases of $\left(\phi_{1}, \phi_{2}, \phi_{3}\right)$. Predictions of $B\left(K^{+} \rightarrow \pi^{+} \nu \bar{\nu}\right)\left(10^{-10}\right), \Delta m_{D}\left(\operatorname{in} 10^{-12} \mathrm{MeV}\right), B\left(K_{L} \rightarrow\right.$ $\left.\pi^{0} \nu \bar{\nu}\right)\left(10^{-10}\right)$ and the CP asymmetry for $B_{d} \rightarrow J / \psi K_{S}$ are added.

\begin{tabular}{c|c|c|c|c|c|c}
\hline \hline$\phi_{1}$ & $\phi_{2}$ & $\phi_{3}$ & $K^{+} \rightarrow \pi^{+} \nu \bar{\nu}$ & $\Delta m_{D}$ & $K_{L} \rightarrow \pi^{0} \nu \bar{\nu}$ & $C_{f}\left(B_{d} \rightarrow J / \psi K_{S}\right)$ \\
\hline $5 \pi / 12$ & $\pi / 3$ & 0 & 2.8 & 1.1 & 11.5 & 0.26 \\
$\pi / 2$ & $\pi / 2$ & $\pi / 6$ & 2.6 & 0.9 & 10.6 & 0.19 \\
$5 \pi / 12$ & $2 \pi / 3$ & $\pi / 3$ & 2.7 & 0.6 & 10.8 & 0.17 \\
$\pi / 2$ & $5 \pi / 6$ & $\pi / 2$ & 2.7 & 0.3 & 11.0 & 0.22 \\
$\pi / 2$ & $\pi$ & $4 \pi / 3$ & 4.8 & 0.1 & 19.8 & 0.38 \\
$7 \pi / 12$ & $7 \pi / 6$ & $3 \pi / 2$ & 4.5 & 0.3 & 18.5 & 0.37 \\
$15 \pi / 8$ & $3 \pi / 2$ & 0 & 5.1 & 0.4 & 19.5 & -0.13 \\
$\pi / 2$ & $3 \pi / 2$ & $11 \pi / 6$ & 4.6 & 0.7 & 19.2 & 0.32 \\
$7 \pi / 4$ & $5 \pi / 3$ & $\pi / 6$ & 5.0 & 0.5 & 18.6 & -0.32 \\
$19 \pi / 12$ & $11 \pi / 6$ & $\pi / 3$ & 5.2 & 0.4 & 18.0 & -0.40 \\
\hline
\end{tabular}


Table 4: Typical solutions for the fourth-generation mixing $\left(s_{w}, s_{v}, s_{u}\right)=$ $\left(0.3 \lambda^{3}, 0.3 \lambda^{2}, \lambda\right)$ in case of $\left(m_{t^{\prime}}, m_{b^{\prime}}\right)=(1200,1170) \mathrm{GeV}$ for the three phases of $\left(\phi_{1}, \phi_{2}, \phi_{3}\right)$. Predictions of $B\left(K^{+} \rightarrow \pi^{+} \nu \bar{\nu}\right)\left(10^{-10}\right), \Delta m_{D}\left(\mathrm{in} 10^{-12} \mathrm{MeV}\right), B\left(K_{L} \rightarrow\right.$ $\left.\pi^{0} \nu \bar{\nu}\right)\left(10^{-10}\right)$ and the $\mathrm{CP}$ asymmetry for $B_{d} \rightarrow J / \psi K_{S}$ are added.

\begin{tabular}{c|c|c|c|c|c|c}
\hline \hline$\phi_{1}$ & $\phi_{2}$ & $\phi_{3}$ & $K^{+} \rightarrow \pi^{+} \nu \bar{\nu}$ & $\Delta m_{D}$ & $K_{L} \rightarrow \pi^{0} \nu \bar{\nu}$ & $C_{f}\left(B_{d} \rightarrow J / \psi K_{S}\right)$ \\
\hline $5 \pi / 12$ & $\pi / 6$ & $23 \pi / 12$ & 1.0 & 0.7 & 3.8 & 0.30 \\
$\pi / 2$ & $\pi / 3$ & 0 & 1.5 & 0.5 & 6.1 & 0.27 \\
$7 \pi / 12$ & $\pi / 2$ & $\pi / 6$ & 1.4 & 0.3 & 5.7 & 0.18 \\
$2 \pi / 3$ & $5 \pi / 6$ & $7 \pi / 12$ & 0.9 & 0.1 & 3.7 & 0.18 \\
$\pi / 2$ & $\pi$ & $3 \pi / 4$ & 1.0 & 0.01 & 3.8 & 0.29 \\
$5 \pi / 12$ & $4 \pi / 3$ & $5 \pi / 3$ & 2.9 & 0.1 & 12.0 & 0.39 \\
$5 \pi / 12$ & $3 \pi / 2$ & $7 \pi / 4$ & 2.1 & 0.3 & 8.5 & 0.36 \\
$5 \pi / 12$ & $5 \pi / 3$ & $23 \pi / 12$ & 2.3 & 0.4 & 9.0 & 0.30 \\
$\pi / 4$ & $11 \pi / 6$ & $\pi / 6$ & 3.1 & 0.4 & 12.8 & 0.14 \\
$\pi / 2$ & $2 \pi$ & $7 \pi / 4$ & 1.1 & 0.5 & 4.2 & 0.35 \\
\hline
\end{tabular}

As can be seen from Tables 2, 3 and 4 for the "maximum" mixing of the fourth generation, the constraints from all the seven quantities considered here except $B\left(K^{+} \rightarrow \pi^{+} \nu \bar{\nu}\right)$ could predict the values $(0.6-5.2) \times 10^{-10}$ for $B\left(K^{+} \rightarrow\right.$ $\left.\pi^{+} \nu \bar{\nu}\right)$, including the values just outside the predictions of the Standard Model, $(0.6-1.5) \times 10^{-10}$, and not so large as the upper part of the measured value of $(0.7-13.9) \times 10^{-10}$. This means that all the seven quantities except the present measurement of $B\left(K^{+} \rightarrow \pi^{+} \nu \bar{\nu}\right)$ have already implied the fourth generation with the mixing as large as $\left(s_{w}, s_{v}, s_{u}\right)=\left(0.8 \lambda^{3}, 0.8 \lambda^{2}, \lambda\right)$ for $m_{t^{\prime}}=400 \mathrm{GeV}$ and so on and that they could predict the quantities of $x_{s}, \Delta m_{D}$ and $B\left(K_{L} \rightarrow \pi^{0} \nu \bar{\nu}\right)$ in the range of values shown in Table 5, which is explained in detail in the next section.

\section{Discussions and conclusions}

We can obtain the following predictions from these maximum mixings; the branching ratio of $K^{+} \rightarrow \pi^{+} \nu \bar{\nu}$ takes a range from the Standard Model(SM) values to the central value of the new measurement as $B=(0.6-5.2) \times 10^{-10}$, 
Table 5: Comparison of $B\left(K^{+} \rightarrow \pi^{+} \nu \bar{\nu}\right), x_{s}\left(B_{s}-\bar{B}_{s}\right.$ mixing $), \Delta m_{D}$ and $B\left(K_{L} \rightarrow\right.$ $\left.\pi^{0} \nu \bar{\nu}\right)$ among the experimental values, Standard Model(SM) predictions and fourgeneration model predictions.

\begin{tabular}{l|c|c|c|c}
\hline \hline & $B\left(K^{+} \rightarrow \pi^{+} \nu \bar{\nu}\right)$ & $x_{s}$ & $\Delta m_{D}(\mathrm{MeV})$ & $B\left(K_{L} \rightarrow \pi^{0} \nu \bar{\nu}\right)$ \\
\hline Experiments & $\left(4.2_{-3.5}^{+9.7) \times 10^{-10}}\right.$ & $>12.8$ & $<1.4 \times 10^{-10}$ & $<5.8 \times 10^{-5}$ \\
SM & $(0.6-1.5) \times 10^{-10}$ & $19-27$ & $\sim 10^{-14}$ & $(1.1-5.0) \times 10^{-11}$ \\
4-generation & $(0.6-5.2) \times 10^{-10}$ & $19-29$ & $(0.01-2.1) \times 10^{-12}$ & $(0.05-22) \times 10^{-10}$ \\
\hline
\end{tabular}

the strength of $B_{s}-\bar{B}_{s}$ mixing is $19 \leq x_{s} \leq 29$, where $x_{s} \equiv \Delta m_{B_{s}} / \Gamma_{B_{s}}, \Gamma_{B_{s}}$ being the total decay rate of $B_{s}$ meson, $\Delta m_{D}$ of $D^{0}-\bar{D}^{0}$ mixing could have a value $(0.01-2.1) \times 10^{-12} \mathrm{MeV}$, extending to about two orders of magnitude larger than the SM prediction $\left(\sim 10^{-14} \mathrm{MeV}[23)\right.$, and the branching ratio of $K_{L} \rightarrow \pi^{0} \nu \bar{\nu}$ takes a range of $(0.05-22) \times 10^{-10}$, ranging from the SM values to the values of two orders of magnitude larger than the SM prediction $\left((1.1-5.0) \times 10^{-11}[2]\right)$. These results are summarized in Table 5 .

The maximum mixing gives an interesting effect on the CP-asymmetry of the decay rates of the "gold-plate" mode of $B_{d}$ meson, $B_{d} \rightarrow J / \psi K_{S}$. The asymmetry is given by

$$
C_{f}=\frac{\Gamma\left(B_{d} \rightarrow J / \psi K_{S}\right)-\Gamma\left(\bar{B}_{d} \rightarrow J / \psi K_{S}\right)}{\Gamma\left(B_{d} \rightarrow J / \psi K_{S}\right)+\Gamma\left(\bar{B}_{d} \rightarrow J / \psi K_{S}\right)},
$$

and it is expressed as 28

$$
\begin{aligned}
C_{f} & =-\frac{x_{d}}{1+x_{d}^{2}} \operatorname{Im} \Lambda=\frac{x_{d}}{1+x_{d}^{2}} \sin 2 \beta, \\
\Lambda & \equiv \sqrt{\frac{M_{12}^{*}}{M_{12}}} \frac{A\left(\bar{B}_{d} \rightarrow J / \psi K_{S}\right)}{A\left(B_{d} \rightarrow J / \psi K_{S}\right)},
\end{aligned}
$$

where $x_{d}$ is the mixing strength of $B_{d}-\bar{B}_{d}$ mixing, $M_{12}$ the off-diagonal element of the mass matrix in $B_{d}-\bar{B}_{d}$ system, $A$ the decay amplitude and $\beta$ is one of the angles of the unitarity triangle. In the Standard Model[29], the quantity $C_{f}$ takes a positive sign as $0.18 \leq C_{f} \leq 0.37$ for $B_{d} \rightarrow J / \psi K_{S}$, resulting from the 
phase range of $0<\phi_{1}<\pi$, which is constrained from the positive sign of the CPviolating parameter $\varepsilon_{K}$. However, in the four-generation model[[], $C_{f}$ can take also a negative sign as $-0.38 \leq C_{f} \leq 0.40$, since the phase $\phi_{1}$ takes the whole range of $0<\phi_{1}<2 \pi$ due to the occurence of the two more new phases $\phi_{2}$ and $\phi_{3}$. For the moment, $\sin 2 \beta$ of eq.(26) has recently been measured to be positive as $\sin 2 \beta=\left(3.2_{-2.0}^{+1.8} \pm 0.5\right)$ by OPAL Collaboration [30] and $\sin 2 \beta=(1.8 \pm 1.1 \pm 0.3)$ by CDF Collaboration [31], which means that $C_{f}$ is positive as in the Standard Model. We should add that although the penguin diagrams could affect the decay amplitude in the four-generation model, they would bring at most several percent change of $C_{f}$.

The unitarity triangle in the Standard Model transforms into unitarity quadrangle in the four-generation model[32]. For the "maximum" mixing obtained here, some of the typical quadrangles are shown in Fig. 4 for $m_{t^{\prime}}=400 \mathrm{GeV}$ and in Fig.5 for $m_{t^{\prime}}=800 \mathrm{GeV}$. The fourth side of the quadrangle, $V_{t^{\prime} d} V_{t^{\prime} b}^{*}$, is of order $\lambda^{4}$, while the other three sides are of order $\lambda^{3}$. The first two quadrangles of Figs.4 and 5 are for positive sign of $C_{f}$. The third ones of Figs. 4 and 5 are for negative sign of $C_{f}$ and are reversed with respect to the base line $V_{c d} V_{c b}^{*}$, since $\phi_{1}>\pi$, where $\phi_{1}$ corresponds to the anti-clockwise angle measured from $V_{c d} V_{c b}^{*}$ to $V_{u d} V_{u b}^{*}$ and $\phi_{3}$ to the anti-clockwise angle from $V_{c d} V_{c b}^{*}$ to $V_{t^{\prime} d} V_{t^{\prime} b}^{*}$. Incidentally, the quadrangles for the solutions with smaller mixing of $\left(s_{w}, s_{v}, s_{u}\right)=\left(\lambda^{4}, \lambda^{3}, \lambda^{2}\right)$ for $m_{t^{\prime}}=400 \mathrm{GeV}$ are given in Fig.6. In this case, the size of the fourth side, $V_{t^{\prime} d} V_{t^{\prime} b}^{*}$, is of order $\lambda^{6}$ and is about $1 / 100$ that of the side $V_{c d} V_{c b}^{*}$ and the quadrangle could not be distinguished from the triangle, and the branching ratio of $K^{+} \rightarrow \pi^{+} \nu \bar{\nu}$ is predicted to be in the range of $(0.6-1.2) \times 10^{-10}$, which agrees with the predictions of the Standard Model. So, if the future measurements of $K^{+} \rightarrow \pi^{+} \nu \bar{\nu}$ show its branching ratio to be in the range of the Standard Model values, the large mixing of the fourth generation obtained here as the "maximum" one will not be allowed. 
Table 6: The same as in Table 2 except that $B_{K}=0.75 \pm 0.05$ and $f_{B}^{2} B_{B}=$ $(0.20 \pm 0.01)^{2} \mathrm{GeV}^{2}$.

\begin{tabular}{c|c|c|c|c|c|c}
\hline \hline$\phi_{1}$ & $\phi_{2}$ & $\phi_{3}$ & $K^{+} \rightarrow \pi^{+} \nu \bar{\nu}$ & $\Delta m_{D}$ & $K_{L} \rightarrow \pi^{0} \nu \bar{\nu}$ & $C_{f}\left(B_{d} \rightarrow J / \psi K_{S}\right)$ \\
\hline$\pi / 4$ & $\pi / 3$ & 0 & 2.2 & 1.8 & 8.7 & 0.24 \\
$\pi / 2$ & $5 \pi / 6$ & $\pi / 2$ & 1.7 & 0.7 & 7.0 & 0.25 \\
$\pi / 2$ & $7 \pi / 6$ & $11 \pi / 12$ & 1.7 & 0.4 & 6.6 & 0.30 \\
\hline
\end{tabular}

Table 7: The same as in Table 3 except that $B_{K}=0.75 \pm 0.05$ and $f_{B}^{2} B_{B}=$ $(0.20 \pm 0.01)^{2} \mathrm{GeV}^{2}$.

\begin{tabular}{c|c|c|c|c|c|c}
\hline \hline$\phi_{1}$ & $\phi_{2}$ & $\phi_{3}$ & $K^{+} \rightarrow \pi^{+} \nu \bar{\nu}$ & $\Delta m_{D}$ & $K_{L} \rightarrow \pi^{0} \nu \bar{\nu}$ & $C_{f}\left(B_{d} \rightarrow J / \psi K_{S}\right)$ \\
\hline$\pi / 2$ & $5 \pi / 6$ & $\pi / 2$ & 2.7 & 0.3 & 11.0 & 0.22 \\
$\pi / 2$ & $\pi$ & $4 \pi / 3$ & 4.8 & 0.1 & 19.8 & 0.38 \\
$15 \pi / 8$ & $3 \pi / 2$ & 0 & 5.1 & 0.4 & 19.5 & -0.13 \\
\hline
\end{tabular}

We should remark that this large mixing of the fourth generation we found here is not due to the fairly large theoretical uncertainties in $B_{K}=0.75 \pm 0.15$ and $f_{B} \sqrt{B_{B}}=(0.20 \pm 0.04) \mathrm{GeV}$. Even if we prescribe to reduce the uncertainties of these quantities by $1 / 3$ to $1 / 4$ keeping the central values as $B_{K}=0.75 \pm 0.05$ and $f_{B} \sqrt{B_{B}}=(0.20 \pm 0.01) \mathrm{GeV}$, we can still find some of the solutions such as listed in Tables 6 and 7 for $m_{t^{\prime}}=400 \mathrm{GeV}$ and $800 \mathrm{GeV}$, respectively.

Summarizing, we find "maximum" mixings of the fourth generation $\left(V_{t^{\prime} d}, V_{t^{\prime} s}, V_{t^{\prime} b}\right) \simeq$ $\left(0.8 \lambda^{3}, 0.8 \lambda^{2}, \lambda\right)$ for $\left(m_{t^{\prime}}, m_{b^{\prime}}\right)=(400,370) \mathrm{GeV},\left(V_{t^{\prime} d}, V_{t^{\prime} s}, V_{t^{\prime} b}\right) \simeq\left(0.5 \lambda^{3}, 0.5 \lambda^{2}, \lambda\right)$ for $\left(m_{t^{\prime}}, m_{b^{\prime}}\right)=(800,770) \mathrm{GeV}$ and $\left(V_{t^{\prime} d}, V_{t^{\prime} s}, V_{t^{\prime} b}\right) \simeq\left(0.3 \lambda^{3}, 0.3 \lambda^{2}, \lambda\right)$ for $\left(m_{t^{\prime}}, m_{b^{\prime}}\right)=$ $(1200,1170) \mathrm{GeV}$, which are consistent with the eight constraints of $\Delta m_{K}, \varepsilon_{K}, B_{d}-$ $\bar{B}_{d}$ mixing, $K^{+} \rightarrow \pi^{+} \nu \bar{\nu}, B_{s}-\bar{B}_{s}$ mixing, $D^{0}-\bar{D}^{0} \operatorname{mixing}, K_{L} \rightarrow \pi^{0} \nu \bar{\nu}$ and $\left(K_{L} \rightarrow \mu \bar{\mu}\right)_{\mathrm{SD}}$. The mass difference $\Delta m_{D}$ from $D^{0}-\bar{D}^{0}$ mixing and the branching ratio of $K_{L} \rightarrow \pi^{0} \nu \bar{\nu}$ could reach the values one to two orders of magnitude larger than the Standard Model predictions, and the CP asymmetry of the decay rates of $B_{d} \rightarrow J / \psi K_{S}$ could take a value of opposite sign to the SM one. Measure- 
ments of $\Delta m_{D}$ and $B\left(K_{L} \rightarrow \pi^{0} \nu \bar{\nu}\right)$ are expected to be done and further data of $B\left(K^{+} \rightarrow \pi^{+} \nu \bar{\nu}\right)$ with more statistics are required.

We are grateful to Takeshi Komatsubara, Minoru Tanaka, Takeshi Kurimoto, Xing Zhi-Zhong, Masako Bando, C.S. Lim, and Morimitsu Tanimoto for helpful discussions.

\section{References}

[1] S. Adler et al., Phys. Rev. Lett. 79, 2204 (1997).

[2] G. Buchalla, A.J. Buras and M.E. Lautenbacher, Rev. Mod. Phys. 68, 1125 (1996), and references therein; G. Buchalla and A.J. Buras, preprint hepph/9901288.

[3] M.K. Gaillard and B.W. Lee, Phys. Rev. D 10, 897 (1974).

[4] T. Inami and C.S. Lim, Prog. Theor. Phys. 65, 297 (1981).

[5] M. Gronau and J. Schechter, Phys. Rev. D31, 1668 (1974); I.I. Bigi, Z. Phys. C27, 303(1985); A.A. Anselm, J.L. Chkareuli, N.G. Uraltsev and T.A. Zhukovskaya, Phys. Lett. B156, 102(1985); X.G. He and S. Pakvasa, Phys. Lett. B156, 236(1985); U. Türke, E.A. Paschos, H. Usler and R. Decker, Nucl. Phys. B285, 313(1985); T. Hayashi, M. Tanimoto and S. Wakaizumi, Prog. Theor. Phys. 75, 353 (1986).

[6] U. Türke, Phys. Lett. B 168, 296 (1986); I.I. Bigi and S. Wakaizumi, Phys. Lett. B 188, 501 (1987); G. Eilam, J.L. Hewett and T.G. Rizzo, Phys. Lett. B 193, 533 (1987).

[7] T. Hasuike, T. Hattori, T. Hayashi and S. Wakaizumi, Mod. Phys. Lett. A 4, 2465 (1989); Prog. Theor. Phys. 83, 265(1990); Phys. Rev. D 41, 1691 (1990); Phys. Rev. D 44, 3582 (1991). 
[8] W.-S. Hou, A. Soni and H. Steger, Phys. Lett. B 192, 441 (1987).

[9] M. Kobayashi and T. Maskawa, Prog. Theor. Phys. 49, 652 (1973).

[10] L. Wolfenstein, Phys. Rev. Lett. 51, 1945 (1983).

[11] Particle Data Group, C. Caso et al., Eur. Phys. J. C3, 1(1998).

[12] W. Adam et al., Phys. Lett. B 414, 382 (1997); R. Barate et al., Eur. Phys. J. C4, 367(1998).

[13] E.M. Aitala et al., Phys. Rev. Lett. 77, 2384 (1996).

[14] M. Weaver et al., Phys. Rev. Lett. 72, 3758 (1994).

[15] G. Bélanger and C.G. Geng, Phys. Rev. D 43, 140 (1991).

[16] A. Alavi-Harati et al., KTeV Collaboration, Phys. Rev. Lett. 83, 22(1999).

[17] G.D. Barr et al., Phys. Lett. B317, 233(1993).

[18] A.J. Buras, TUM-HEP-349/99, hep-ph/9905437.

[19] G. Buchalla and A.J. Buras, Nucl. Phys. B 398, 285 (1993); Nucl Phys. B 400, 225 (1993).

[20] G. Buchalla and A.J. Buras, Nucl. Phys. B 412, 106 (1994).

[21] D. Rein and L.M. Sehgal, Phys. Rev. D 39, 335 (1989).

[22] K.S. Babu, X.-G. He, X.-Q. Li and S. Pakvasa, Phys. Lett. B 205, 540 (1988).

[23] A. Datta and D. Kumbhakar, Z. Phys. C 27, 515 (1985).

[24] L. Wolfenstein, Phys. Lett. B 164, 170 (1985); J. Donoghue, E. Golowich, B.R. Holstein and J. Trampetić, Phys. Rev. D 33, 179 (1986). 
[25] L.S. Littenberg, Phys. Rev. D 39, 3322 (1989).

[26] G. Buchalla and G. Isidori, Phys. Lett. B440, 170(1998).

[27] Particle Data Group, C. Caso et al., Eur. Phys. J. C3, 1(1998), p97-98.

[28] A.B. Carter and A.I. Sanda, Phys. Rev. D 23, 1567 (1981); I.I. Bigi and A.I. Sanda, Nucl. Phys. B 193, 85 (1981).

[29] I. Dunietz and J.L. Rosner, Phys. Rev. D 34, 1404 (1986).

[30] K. Akerstaff et al., Eur. Phys. J. C5, 379(1998).

[31] F. Abe et al., Phys. Rev. Lett. 81, 5513(1998).

[32] Y. Nir and H.R. Quinn, in B Decays, edited by S. Stone (world Scientific, Singapore, 1992). 


\section{Figure captions}

Fig.1. $W-W$ box diagram for $K_{L}-K_{S}$ mass difference in the four-generation model.

Fig.2. $W-W$ box and $Z^{0}$-penguin diagrams for $K^{+} \rightarrow \pi^{+} \nu \bar{\nu}$.

Fig.3. The dominant $W-W$ box diagram for $D^{0}-\bar{D}^{0}$ mixing in the fourgeneration model.

Fig.4. Typical examples of the unitarity quadrangle for $\left(s_{w}, s_{v}, s_{u}\right)=\left(0.8 \lambda^{3}, 0.8 \lambda^{2}, \lambda\right)$ in case of $\left(m_{t^{\prime}}, m_{b^{\prime}}\right)=(400,370) \mathrm{GeV}$. (a) $\phi_{1}=\frac{\pi}{2}, \phi_{2}=2 \pi, \phi_{3}=\frac{7}{4} \pi ; B\left(K^{+} \rightarrow\right.$ $\left.\pi^{+} \nu \bar{\nu}\right)=1.6 \times 10^{-10}, C_{f}\left(B_{d} \rightarrow J / \psi K_{S}\right)=0.34$, (b) $\phi_{1}=\frac{\pi}{2}, \phi_{2}=\frac{5}{6} \pi, \phi_{3}=$ $\frac{\pi}{2} ; B\left(K^{+} \rightarrow \pi^{+} \nu \bar{\nu}\right)=1.7 \times 10^{-10}, C_{f}\left(B_{d} \rightarrow J / \psi K_{S}\right)=0.25,(\mathrm{c}) \phi_{1}=\frac{13}{8} \pi, \phi_{2}=$ $\pi, \phi_{3}=\frac{3}{4} \pi ; B\left(K^{+} \rightarrow \pi^{+} \nu \bar{\nu}\right)=2.7 \times 10^{-10}, C_{f}\left(B_{d} \rightarrow J / \psi K_{S}\right)=-0.35$.

Fig.5. Typical examples of the unitarity quadrangle for $\left(s_{w}, s_{v}, s_{u}\right)=\left(0.5 \lambda^{3}, 0.5 \lambda^{2}, \lambda\right)$ in case of $\left(m_{t^{\prime}}, m_{b^{\prime}}\right)=(800,770) \mathrm{GeV}$. (a) $\phi_{1}=\frac{\pi}{2}, \phi_{2}=\frac{3}{2} \pi, \phi_{3}=\frac{11}{6} \pi ; B\left(K^{+} \rightarrow\right.$ $\left.\pi^{+} \nu \bar{\nu}\right)=4.6 \times 10^{-10}, C_{f}\left(B_{d} \rightarrow J / \psi K_{S}\right)=0.32$, (b) $\phi_{1}=\frac{5}{12} \pi, \phi_{2}=\frac{2}{3} \pi, \phi_{3}=$ $\frac{\pi}{3} ; B\left(K^{+} \rightarrow \pi^{+} \nu \bar{\nu}\right)=2.7 \times 10^{-10}, C_{f}\left(B_{d} \rightarrow J / \psi K_{S}\right)=0.17,(\mathrm{c}) \phi_{1}=\frac{19}{12} \pi, \phi_{2}=$ $\frac{11}{6} \pi, \phi_{3}=\frac{\pi}{3} \pi ; B\left(K^{+} \rightarrow \pi^{+} \nu \bar{\nu}\right)=5.2 \times 10^{-10}, C_{f}\left(B_{d} \rightarrow J / \psi K_{S}\right)=-0.40$.

Fig.6. Typical examples of the unitarity quadrangle for $\left(s_{w}, s_{v}, s_{u}\right)=\left(\lambda^{4}, \lambda^{3}, \lambda^{2}\right)$ in case of $\left(m_{t^{\prime}}, m_{b^{\prime}}\right)=(400,370) \mathrm{GeV}$. (a) $\phi_{1}=\frac{\pi}{2}, \phi_{2}=\frac{\pi}{6}, \phi_{3}=\frac{7}{4} \pi ; B\left(K^{+} \rightarrow\right.$ $\left.\pi^{+} \nu \bar{\nu}\right)=0.94 \times 10^{-10}, C_{f}\left(B_{d} \rightarrow J / \psi K_{S}\right)=0.30$, (b) $\phi_{1}=\frac{\pi}{4}, \phi_{2}=\frac{\pi}{6} \pi, \phi_{3}=$ $\frac{3}{4} \pi ; B\left(K^{+} \rightarrow \pi^{+} \nu \bar{\nu}\right)=0.89 \times 10^{-10}, C_{f}\left(B_{d} \rightarrow J / \psi K_{S}\right)=0.28,(\mathrm{c}) \phi_{1}=\frac{\pi}{4}, \phi_{2}=$ $\frac{\pi}{3}, \phi_{3}=\frac{5}{4} \pi ; B\left(K^{+} \rightarrow \pi^{+} \nu \bar{\nu}\right)=1.0 \times 10^{-10}, C_{f}\left(B_{d} \rightarrow J / \psi K_{S}\right)=0.29$. 


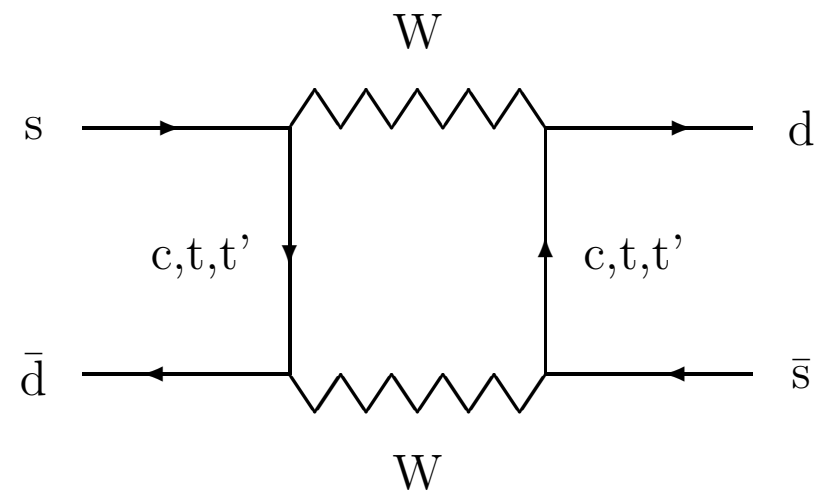

Figure 1: 

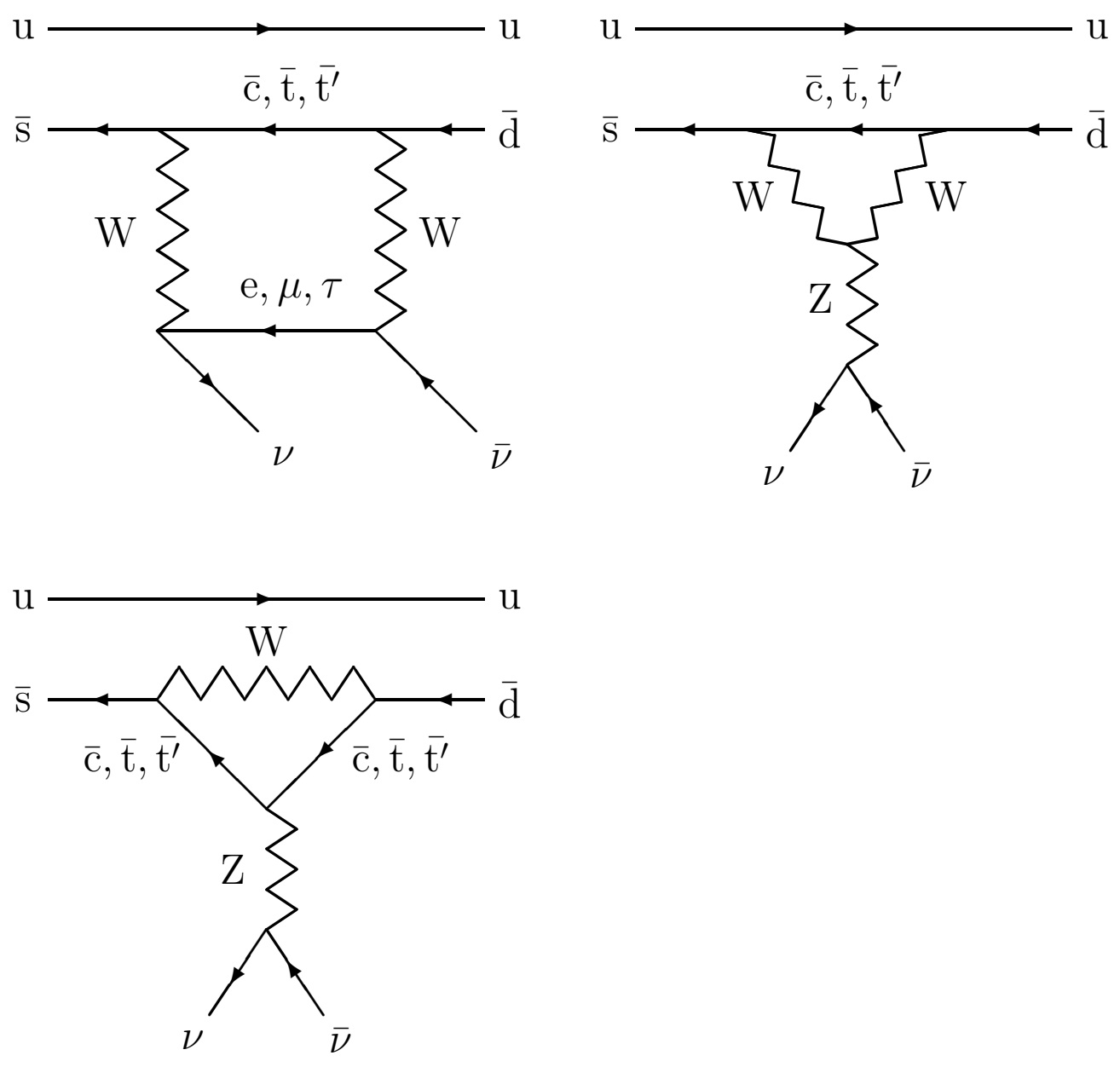

Figure 2: 


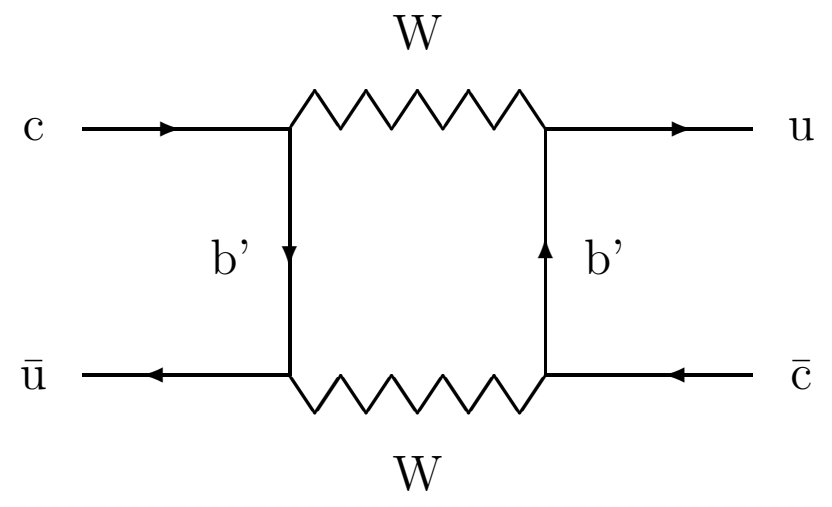

Figure 3: 
(a)

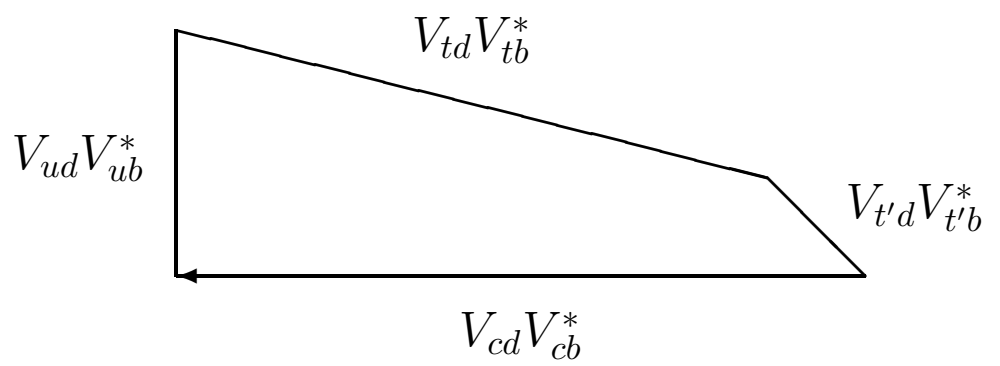

(b)

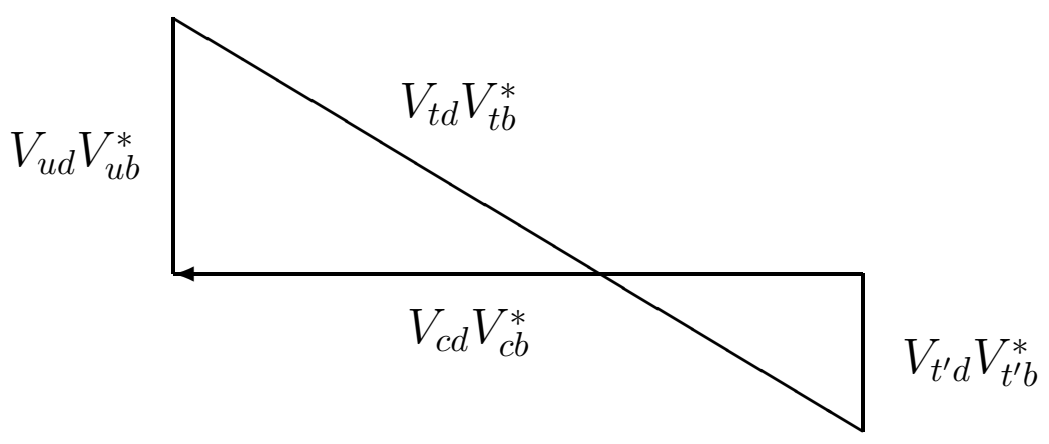

(c)

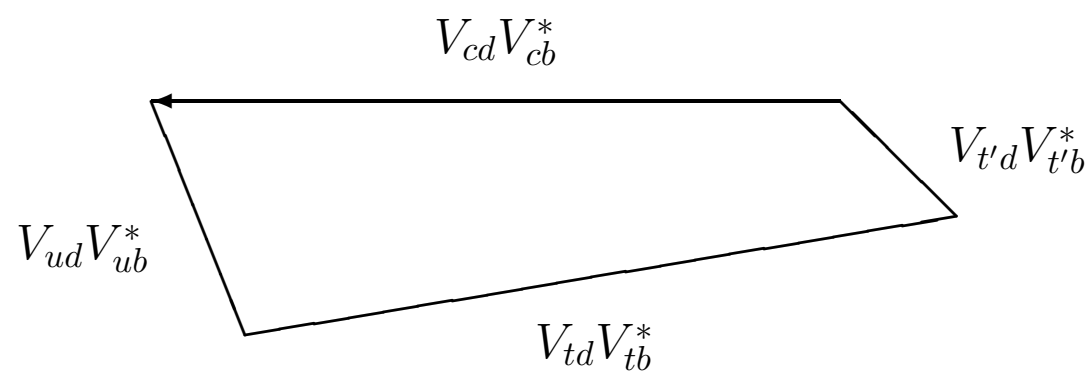

Figure 4: 
(a)

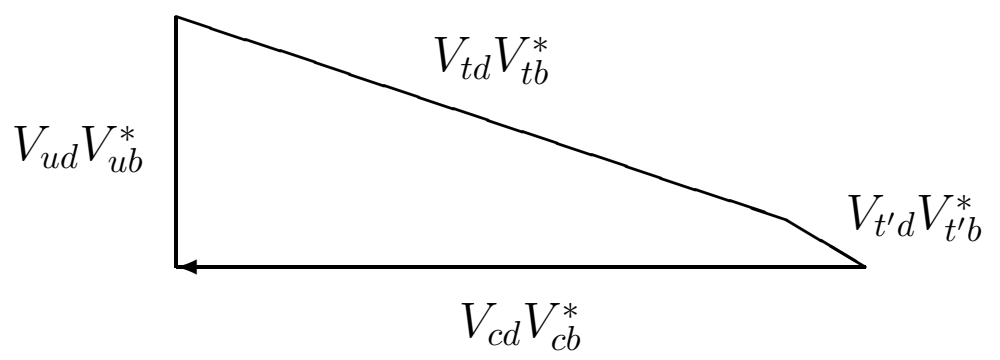

(b)

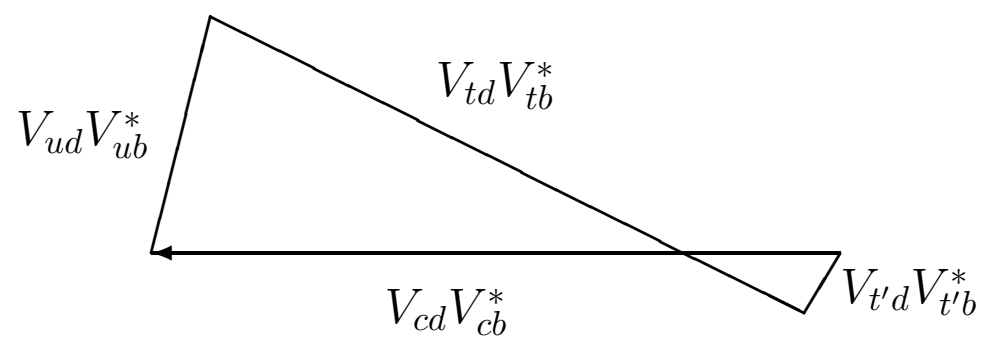

(c)

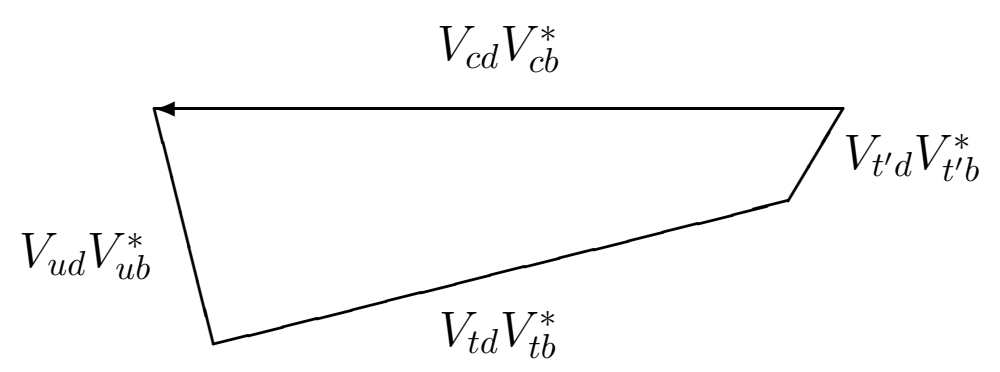

Figure 5: 
(a)

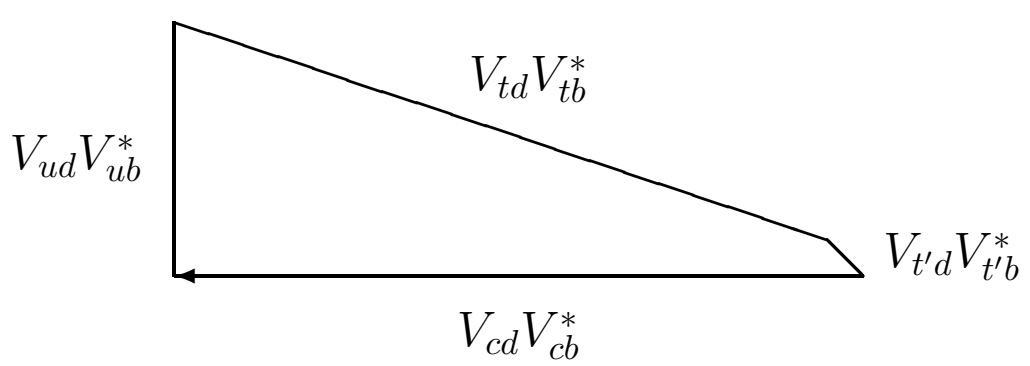

(b)

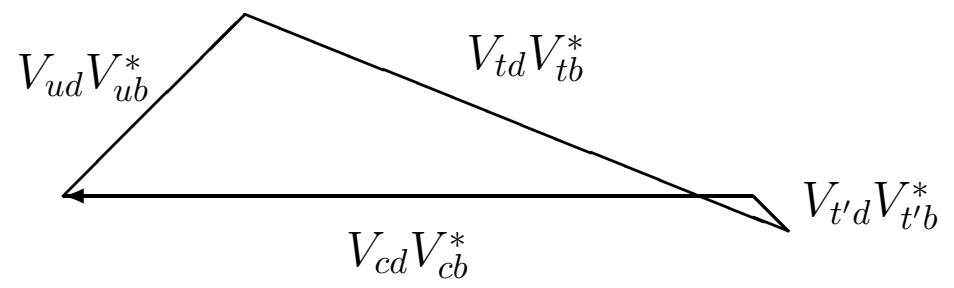

(c)

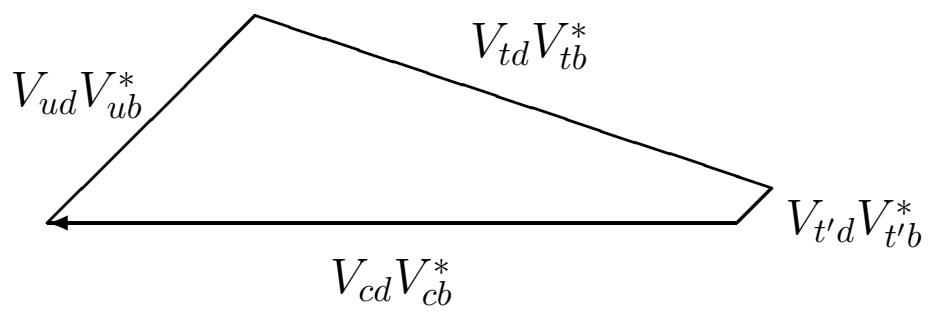

Figure 6: 